\title{
Homeomorphism Groups and the Topologist's Sine Curve by
}

\author{
Jan J. DIJKSTRA and Rachid TAHRI \\ Presented by Czestaw BESSAGA
}

Summary. It is shown that deleting a point from the topologist's sine curve results in a locally compact connected space whose autohomeomorphism group is not a topological group when equipped with the compact-open topology.

If $X$ is a Hausdorff topological space then we let $\mathcal{H}(X)$ denote the group of autohomeomorphisms of $X$ equipped with the compact-open topology. If $A, B \subset X$ then we define $[A, B]=\{h \in \mathcal{H}(X): h(A) \subset B\}$ and we recall that the topology on $\mathcal{H}(X)$ is generated by the subbasis $\{[K, O]$ : $K$ compact and $O$ open in $X\}$. If $X$ is locally compact, then composition is continuous on $\mathcal{H}(X)$ and if $X$ is compact, then also the inverse operation is continuous, thus $\mathcal{H}(X)$ is a topological group; see Arens [1]. The Cantor set with a point removed is the standard example of a locally compact metric space such that the inverse operation on $\mathcal{H}(X)$ is discontinuous; see for instance [3. It is a classic theorem of Arens [1] that if $X$ is locally compact and locally connected then $\mathcal{H}(X)$ is a topological group. Dijkstra improved on this result in [3] with the following theorem. A continuum is a compact connected space.

THEOREM 1. If every point in a Hausdorff space $X$ has a neighbourhood that is a continuum then $\mathcal{H}(X)$ is a topological group.

It is an open problem to determine exactly for which locally compact (metric) spaces $X$ the space $\mathcal{H}(X)$ is a topological group. Note that Theorem 1 states that it is sufficient that every point has a neighbourhood that is both compact and connected. A natural question that arises is whether this

2010 Mathematics Subject Classification: 54H11, 57S05.

Key words and phrases: topologist's sine curve, homeomorphism group, topological group, compact-open topology. 
condition can be weakened to "every point has a compact neighbourhood and a connected neighbourhood". A variation on this question was posed to us by Fredric Ancel, namely whether local compactness together with connectedness of the whole space is sufficient. We will show that neither is the case by presenting a counterexample. Our counterexample is based on a classic space, namely the topologist's sine curve, which means that we reveal a new facet of this interesting space.

The topologist's sine curve $S$ is a compact subspace of the plane $\mathbb{R}^{2}$ that is the union of the following two sets:

$$
A=\{(0, y):-1 \leq y \leq 1\} \quad \text { and } \quad B=\{(x, \sin (1 / x)): 0<x \leq 1 / \pi\} .
$$

Note that $B$ is homeomorphic to a half-open interval and that the line segment $A$ is contained in the closure of $B$, so that $S$ is a connected space. However, it is not hard to see that there is no path in $S$ that connects points of $A$ with points of $B$, so $S$ is not path connected. Also $S$ is not locally connected at points of $A$. As such $S$ is a popular counterexample in topology and can be found in many topology texts; see for instance [6. p. 137], [2, Examples 5.2.2 and 5.5.3], or [5, Example 24.7].

Let $p=(0,1)$ be the upper endpoint of the arc $A$. The space we are interested in is $S_{p}=S \backslash\{p\}$. This space is obviously locally compact as an open subspace of $S$ and it is connected by the same argument as used for $S$.

TheOREM 2. The inverse operation on $\mathcal{H}\left(S_{p}\right)$ is not continuous.

Proof. We will construct a sequence $h_{1}, h_{2}, \ldots$ in $\mathcal{H}\left(S_{p}\right)$ that converges to the identity function $e$ and such that the inverses $h_{1}^{-1}, h_{2}^{-1}, \ldots$ do not converge to $e$. Let $\rho$ denote the standard euclidean metric on $\mathbb{R}^{2}$.

Let $n$ be a natural number. Put $r_{n}=2 \pi n+\frac{1}{2} \pi$ and note that $\sin r_{n}=1$ and $\left(1 / r_{n}, 1\right) \in S$. Define $\alpha_{n} \in \mathcal{H}([\pi, \infty))$ by

(1) $\alpha_{n}$ is the identity on $\left[\pi, r_{n}-2^{-n}\right]$;

(2) $\alpha_{n}(t)=t+2 \pi$ for $t \geq r_{n}+2^{-n}$;

(3) $\alpha_{n}$ maps $\left[r_{n}-2^{-n}, r_{n}+2^{-n}\right]$ linearly onto $\left[r_{n}-2^{-n}, r_{n}+2 \pi+2^{-n}\right]$.

Note that it follows from (3) that the midpoint $r_{n}$ of the first interval is mapped onto the midpoint $r_{n}+\pi$ of the second interval. We define the bijection $h_{n}: S \rightarrow S$ by letting $h_{n}$ be the identity on $A$ and on $B$,

$$
h_{n}(1 / t, \sin t)=\left(1 / \alpha_{n}(t), \sin \alpha_{n}(t)\right) \quad \text { for } t \geq \pi .
$$

Note that if $t \geq r_{n}+2^{-n}$ then $h_{n}(1 / t, \sin t)=(1 /(t+2 \pi)$, sin $t)$ so the distance between $(1 / t, \sin t)$ and its image $h_{n}(1 / t, \sin t)$ is less than $1 / t$, which is in turn less than $1 / 2 \pi n$. Thus $h_{n}$ is continuous at points of $A$. Since the restriction of $h_{n}$ to the open set $B$ is obviously continuous we see that $h_{n}$ is continuous and thus a homeomorphism by compactness. Since $h_{n}(p)=p$ we can also regard $h_{n}$ as an element of $\mathcal{H}\left(S_{p}\right)$. 


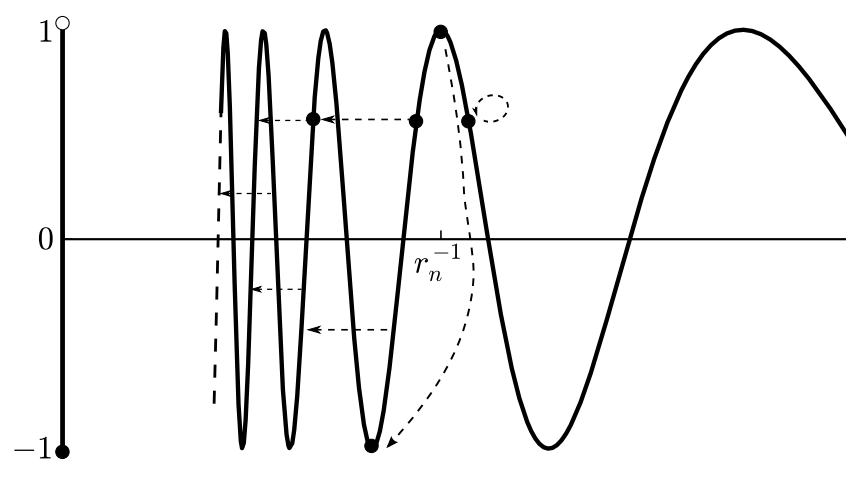

Define the compactum $C=\left\{(x, y) \in S_{p}: y=-1\right\}$ and the open set $U=\left\{(x, y) \in S_{p}: y<1\right\}$ and note that $e \in[C, U]$. For $n \in \mathbb{N}$ we have $h_{n}\left(1 / r_{n}, 1\right)=\left(1 /\left(r_{n}+\pi\right),-1\right)$ thus the inverse of $h_{n}$ is not in $[C, U]$. We may conclude that $h_{1}^{-1}, h_{2}^{-1}, \ldots$ do not converge to $e$.

To show that $\lim _{n \rightarrow \infty} h_{n}=e$ in $\mathcal{H}\left(S_{p}\right)$ let $K$ and $O$ be compact respectively open in $S_{p}$ with $e \in[K, O]$. Thus $K \subset O$ and the distance $d$ between the compacta $K$ and $S \backslash O$ is positive. Since $p \notin O$ and thus $p \notin K$ there is a positive $\varepsilon$ so that $[0, \varepsilon] \times[1-\varepsilon]$ is disjoint from $K$. Select a natural number $N$ such that $\sin \left(\pi / 2-2^{-N}\right)>1-\varepsilon$ and $1 / 2 \pi N$ is less than both $d$ and $\varepsilon$. Let $n \geq N$ and consider $h_{n}$ and a point $(x, y)$ in $K$. If $(x, y) \in A$ or $x \geq 1 /\left(r_{n}-2^{-n}\right)$, then $h_{n}(x, y)=(x, y) \in O$. If $0<x \leq 1 /\left(r_{n}+2^{-n}\right)$ then $\rho\left((x, y), h_{n}(x, y)\right)<1 / 2 \pi n<d$, thus $h_{n}(x, y) \in O$. If $1 /\left(r_{n}+2^{-n}\right)<x<$ $1 /\left(r_{n}-2^{-n}\right)$ then

$$
y=\sin (1 / x) \geq \sin \left(\pi / 2-2^{-n}\right) \geq \sin \left(\pi / 2-2^{-N}\right)>1-\varepsilon
$$

and $x<1 / 2 \pi n \leq 1 / 2 \pi N<\varepsilon$. Thus $(x, y)$ cannot be in $K$ in this case. We have shown that $h_{n} \in[K, O]$ for $n \geq N$ and hence that $\lim _{n \rightarrow \infty} h_{n}=e$ in $\mathcal{H}\left(S_{p}\right)$.

If we identify in $S$ the endpoint $(0,-1)$ of $A$ with the endpoint $(1 / \pi, 0)$ of $B$ then the resulting quotient space is path connected and known as the Warsaw circle; see [4, p. xiii]. If we also delete the point $p$ from the Warsaw circle, then we get a path connected and locally compact space whose homeomorphism group is not a topological group.

\section{References}

[1] R. Arens, Topologies for homeomorphism groups, Amer. J. Math. 68 (1946), 593-610.

[2] F. H. Croom, Principles of Topology, Saunders, Philadelphia, 1989.

[3] J. J. Dijkstra, On homeomorphism groups and the compact-open topology, Amer. Math. Monthly 112 (2005), 910-912. 
[4] S. Mardešić and J. Segal, Shape Theory, North-Holland, Amsterdam, 1982.

[5] J. R. Munkres, Topology, 2nd ed., Prentice Hall, Upper Saddle River, 2000.

[6] L. A. Steen and J. A. Seebach, Counterexamples in Topology, Holt, Rinehart and Winston, New York, 1970.

Jan J. Dijkstra, Rachid Tahri

Faculteit der Exacte Wetenschappen / Afdeling Wiskunde

Vrije Universiteit

De Boelelaan 1081a

1081 HV Amsterdam, The Netherlands

E-mail: dijkstra@cs.vu.nl

rtahri1981@gmail.com 